\title{
Optimization design of papery traffic signs rod based on the research of Midas Civil
}

\author{
Jiangzhu Long ${ }^{1, a}$, Bin Shi ${ }^{2, b}$, Xiaofeng Jiang ${ }^{3, c}$ \\ ${ }^{123}$ Yango College, Fuzhou Fujian

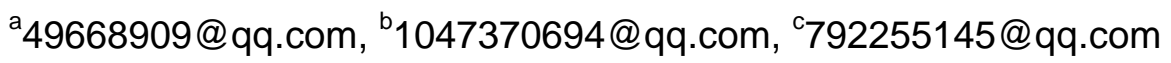

\begin{abstract}
Keywords: Midas Civil; Traffic signs rod; The structure of the paper; Optimization design
Abstract: In the Seventh College Students Structural-Design Competition of Fujian province,the topic is structure design, manufacture and test for Paper traffic signs rod.In this context, seeking a kind of light weight, high strength of traffic signs rod paper structure model become a focus.Due to this,the study first conducted preliminary design of model programs. Then using the advanced civil structure analysis system Midas Civil software to mathematical-modeling, load-simulating and stress analysis for paper traffic sign rod. Making model and through the loading of entity model to find the weak spot. Finally through many times of optimization design to form a kind of high-quality traffic sign rod structure model.
\end{abstract}

\section{Introduction}

As we know, traffic signs rod mainly bears the vertical load of self-weight and the horizontal load under natural wind.According to the title of Seventh College Students Structural-Design Competition:structure design, manufacture and test for Paper traffic signs rod, seeking a kind of light weight, high strength of traffic signs rod paper structure model become a focus. This paper using the Midas Civil software to modeling, analyze and calculating and dynamic simulating. In this way, the waste of material due to repeated modeling and loading could be better avoided.And providing valuable analysis results of paper modeling and optimization for traffic signs rod.

\section{The preliminary design of model program}

The basic requirements of 7th College Students Structural-Design Competition rules are as follows:

As shown in Fig.1, the gripping surface center (i.e., the halfway point of the column bottom width b) is the coordinate origin $\mathrm{O}$. $\mathrm{OA} \geq 800 \mathrm{~mm}$, the coordinates of A point on the $\mathrm{X}$ axis should be $450 \mathrm{~mm}$ or more, in the Y coordinates should be $450 \mathrm{~mm}$ or more. The maximum size for the bottom of the model is limited in less than $200 \mathrm{~mm} \times 200 \mathrm{~mm}$ square, the model can be fixed on the substrate by clamping device.

The bottom of the structure model must provide at least $100 \mathrm{~mm}$ wide (X direction) of the gripping surface, and the height of $\mathrm{Y}$ direction should be at lest $5 \mathrm{~mm}$. The width direction(X direction) of clamping steel plate must be coincidence with central axis of gripping surface,and the clamping device in the longitudinal direction is the $\mathrm{Z}$ direction. Structural components existing above gripping surface is allowed,and must pay attention to the limitation of clamping device (Make sure that there has sufficient space of clamping device for installation). 


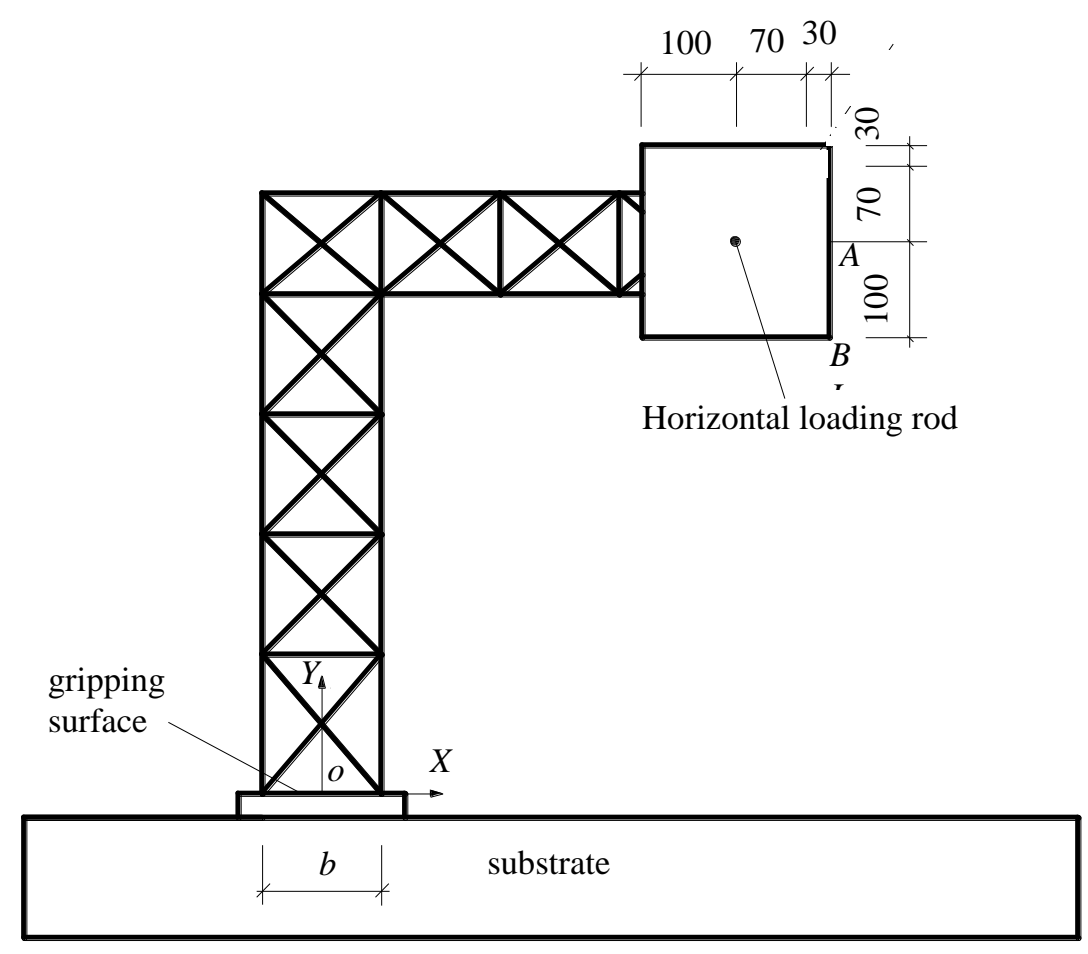

Fig.1. Diagram 1 model size

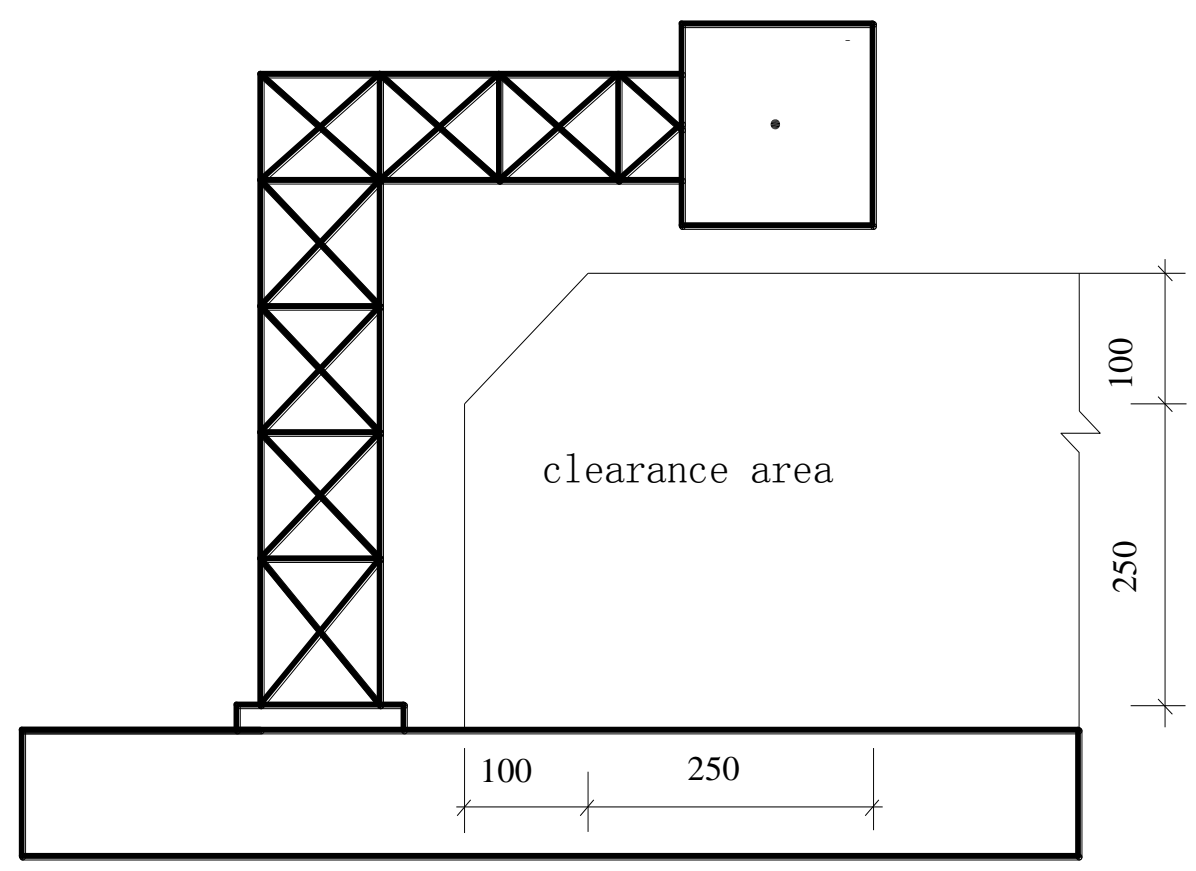

Fig.2. Diagram 2model size

As shown in Fig.2, Clearance area is not allowed to have any component in the Z-direction.

According to request of contest rules, the final design of model program is fromed by repeatedly simulation experiments, as shown in Fig.3.

\section{For papery traffic signs rod modeling}

Steps of using Midas Civil to establish mathematical model: Establish units and nodes $\rightarrow$ Define the material and sectional properties $\rightarrow$ Define load and load groups $\rightarrow$ Define boundaries $\rightarrow$ Define construction stage $\rightarrow$ Run structural analysis. 
Establish units and nodes: Creat a new project in Midas Civil, establish nodes according to each component location of the model,then connecting nodes to form structural units according to the design model.The nodes can be set up on the support parts of the rod,parts of loading concentrated load and the junction of two or more beam elements.

Define the material and sectional properties: Define all the materials and the cross section of the model structure. First of all, according to the requirements of the 7th college students structural design competition in fujian province, participants use white cardboard as the main material.Therefore, when defining material properties, using user-defined mode, and the material elastic modulus of single component should be defined as $0.0569 \times 103 \mathrm{~N} / \mathrm{mm}$. Then based on the section properties of model design program, diameters of $6 \mathrm{~mm}$ and $10 \mathrm{~mm}$ round rod and sectional dimension of $10 \mathrm{~mm} \times 10 \mathrm{~mm}$ and $10 \mathrm{~mm} \times 6 \mathrm{~mm}$ square rod were adopted.

Define load and load groups: In the process of loading,the model of traffic sign rod bears gravity constant load in the vertical direction. The competition use a $1.275 \mathrm{~kg}$ weight to instead of the load. The weight placement center of gravity in the design is located on the 15th node position,so the vertical load point is node 15.It bears wind load in the horizontal direction, and the transverse uniform centralized location of horizontal wind load is located on the 11th node position, so the lateral load point is node 11, as shown in Fig.4.

Define boundaries: Setting the boundary conditions to the beam element and node according to actual condition, and defining the boundary conditions as boundary group.Boundary conditions are defined in four feet point of substrate in this model as a general bearing. Its translational and rotational motion were all restricted. In other words, the structure and foundation were solidified.The boundary conditions as shown in Fig.4.

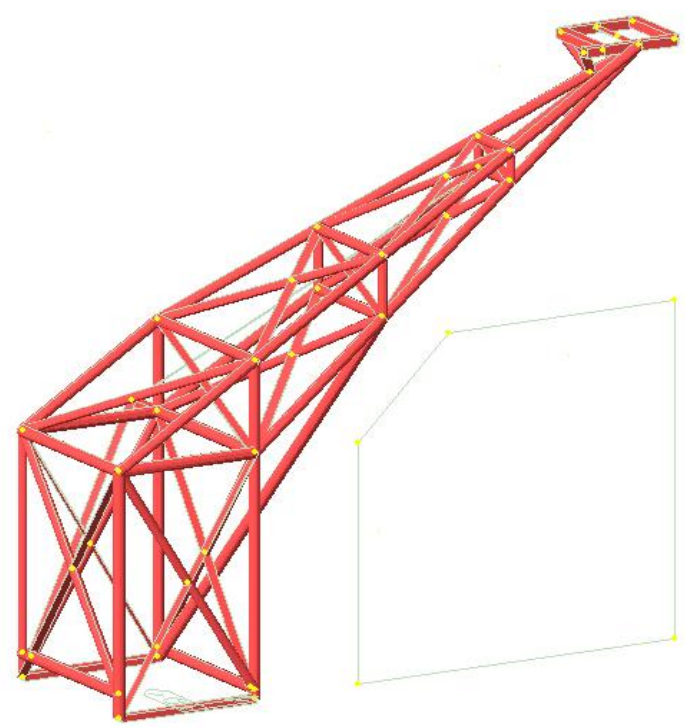

Fig.3. Model design drawings

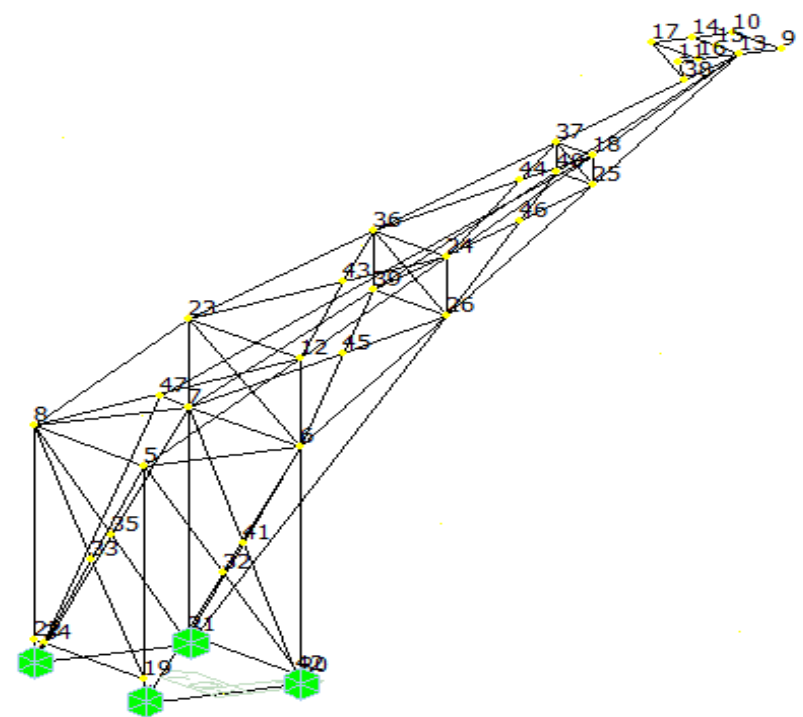

Fig.4. For papery traffic signs rod Midas modeling

Load simulation of construction stage: Appling static load to the model and study with finite-element analysis.Load simulation is divided into two times.

The first load. Applying a load combination in node 11 and node 15 at the same time, $25 \mathrm{~N}$ horizontal load to node 11 and $-12.75 \mathrm{~N}$ vertical load to node 15 , as shown in Fig.3. The purpose of loading is to test stiffness.According to the requirements of competition, the model cannot be overall instability, collapse or horizontal displacement more than allowable values $(90 \mathrm{~mm})$ in the process of the loading for 15 seconds.If the model meet the requirements means it has enough stiffness to the next phase of loading.

Simulate the load bearing capacity. Applying a load combination in node 11 and node 15 at the same time, $75 \mathrm{~N}$ horizontal load to node 11 and $-12.75 \mathrm{~N}$ vertical load to node 15 , as shown in Fig.4.As the requirements of competition,on the basis of the previous load for secondary load. The maximum total load of the model should less than $7.5 \mathrm{~kg}$. In the process of two separate loading for 15 seconds, 
the model cannot be overall instability, collapse or horizontal displacement more than allowable values $(90 \mathrm{~mm})$. Then the structure model satisfies the requirement of maximum bearing capacity.

\section{Analysis of calculation results}

In order to understand and master the mechanical characteristics of actual model better,the program applies the finite element analysis software Midas Civil for mathematical modeling, load-simulating and stress analysis of the deformation.Through analyzing the stress distribution of paper traffic signs rod, the results such as displacement and reaction force can be referred to optimize the model. Repeated optimization aimed at seeking a kind of light weight, high strength structure model of traffic signs rod made by paper.

Paper structure model of traffic signs rod generally use beam element for modeling.There are 46 nodes and 99 beam element in total.The displacement of each node in the model is shown in Fig.5 and Fig.6.The largest displacement of traffic sign rod is shown in Fig.6.

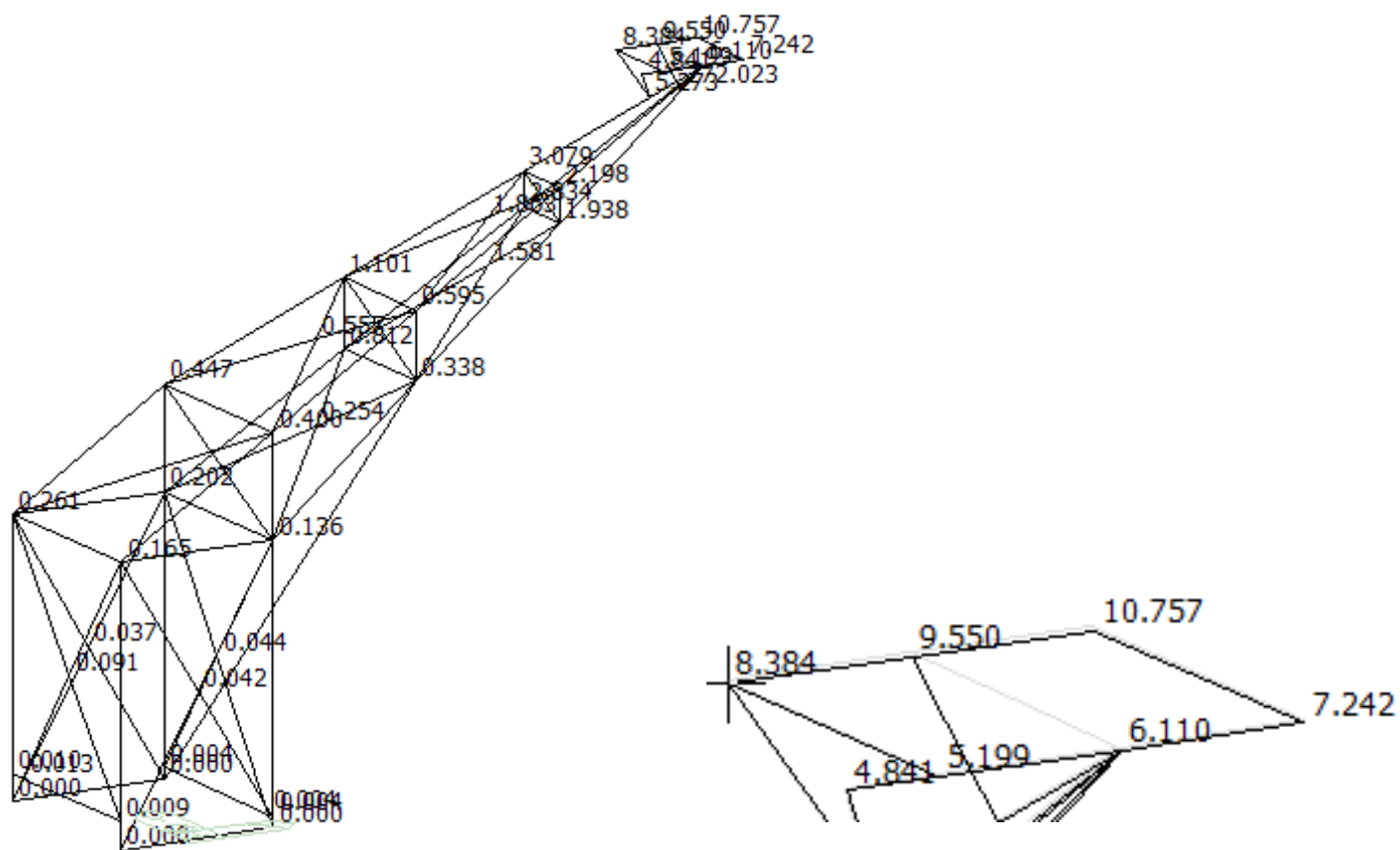

Fig.5. The vertical displacement of

Fig.6. The main node of the vertical each node of Traffic signs in the $\operatorname{model}(\mathrm{mm})$ displacement of the figure in Structure model (mm)

The unit of maximum lateral displacement and biggest stress can be calculated by the software. Therefore, it is better to achieve the goal of practicability and economy by strengthen the weak rod and weaken the rod which stress less. Through using MIDAS Civil finite element analysis, the whole stress distribution of the model and the capacity of each member can be obtained.According to the structure stress conditions to determine the design length of rod and the type of cross section. By strengthening the weak part and reducing material waste to make full use of the material to achieve a better structure.

\section{Conclusions}

Using Midas Civil software to establish the model of cantilever traffic signs rod, appling load in both horizontal load and vertical,then analyzing the force condition of the model under the load.In such a holo-authentic simulation environment, the end of the cantilever structure which is the weak load-bearing point of the rod will produces horizontal offset and vertical displacement under the condition of load application. The change of displacement drives the cantilever rod into bending and torsion which leads to the destruction of the model.So it must be the focus of the optimization design. 
Midas Civil software provides a convenient condition for structure design,so we can predict the damage and the weak load-bearing point of the model through the model established by Midas Civil software. On the basis of analyzing and optimizing the model repeatedly, a mechanical model will be established under the optimal condition, and Formed the best model design scheme.

It is a bold and innovative idea to apply Midas Civil software to the structure model optimal design of traffic sign rod which is made by paper.Software application for the structure modeling of traffic sign rod and optimizing provides valuable analysis results.

\section{Acknowledgements}

College students' innovative entrepreneurial training program of fujian province (201435108024).

\section{References}

[1] The organizing committee of the 7th college students structural design competition in fujian province. Detailed rules for the 7th college students structural design competition in fujian province, 2014(1).

[2] Ling-xi Qian. Optimum design of engineering structure[M]. Beijing: Science Press. 2011.

[3] Yu-chuan Jiang,Chang-bin Fu,Hui-qun Yan. MIDAS application in structural calculation[M]. Beijing: Chemical Industry Press. 2011.

[4] The ministry of transport of the People's Republic of China. JTG D82-2009 The specification of Highway traffic signs and markings set [S]. Beijing:China communications press,2009.

[5] Zhi-gang Wang,Jian-chun Mu,Zhi-qin Long,Hui-feng Xi. The experiment study of paper material mechanical properties under static load [J]. Guangxi Light Industry.2009,25(6).

[6] Yong-sheng Wu,Yun Xie. Midas/civil software application in the temporary structure calculation[J]. Fujian Architecture \& Construction,2012(4).

[7] Lei Wang,Pei-cheng Yang,Jia Wang. Midas/civil application in steel pipe pile bracket design[J]. Transportation Science \& Technology,2010(4).

[8] Cheng-li Wang. Analysis of structure optimization design[J]. Low carbon world,2014(6).

[9] Zhen-hu Du,Guang-zhong Zhong,Hua-wan Hu. Midas application in steel combined section calculation[J]. Sichuan Architecture,2010(1).

[10] Ting-jun Zhou,Guo-kun Fan. Discussion on building structure optimization[J]. World Garden,2014(5).

[11] Bao-xiang Xia,Han-zhong Yang,Ning Jia. The design and application of a new type portal frame for traffic signs [J].Highway,2008(10).

[12] Qiang Liu,Xiao-long Mao. Midas/Civil in the application of the steel pier structure optimization design[J]. China Water Transport,2014(6). 